\title{
Resonance in Upconversion Excitation
}

Karl W. Krämer

Department of Chemistry and Biochemistry, University of Bern, Freiestrase 3, 3012 Bern, Switzerland

Email address: karl.kraemer@dcb.unibe.ch

\begin{abstract}
Upconversion (UC) processes are discussed on the examples of excited state absorption (ESA) in $\mathrm{LaCl}_{3}: 1 \% \mathrm{Er}^{3+}$ for $980 \mathrm{~nm}$ excitation and energy transfer upconversion (ETU) in $\beta-\mathrm{NaYF}_{4}: 20 \% \mathrm{Er}^{3+}$ for $1523 \mathrm{~nm}$ excitation. The crucial importance of resonance is demonstrated for one- and two-color excitations in $\mathrm{LaCl}_{3}: \mathrm{Er}$. For ESA the excitation spectra are convolutions of the individual excitation steps and the UC luminescence intensity shows a linear dependence on the excitation power for each step. For one-color excited ESA and ETU in the low power limit, the exponent of the power dependence is equal to the number of excitation steps. The exponent is reduced due to saturation effects for high power excitation. The ETU excitation spectra resemble the ground state absorption as shown for $\beta$-NaYF $4: E r$. Upon broad-band excitation by sub-Si-band-gap infrared light $\beta$-NaYF4:Er shows UC luminescence mainly from the ${ }^{4} I_{11 / 2}$ state around $980 \mathrm{~nm}$ which efficiently excites a Si solar cell. This Er UC luminescence is two orders of magnitude more intense than that of other rare earth ions under the same experimental conditions.
\end{abstract}

Keywords: upconversion, excitation, $\mathrm{LaCl}_{3}: \mathrm{Er}, \beta-\mathrm{NaYF}_{4}: \mathrm{Er}$

Published in:

Journal of Luminescence 189 (2017) 78 - 83.

DOI: 10.1016/j.jlumin.2016.11.056 


\section{Introduction}

The term upconversion (UC) comprises mechanisms where the absorption of several photons of lower energy results in the emission of one photon of higher energy. After initial work mainly by the group of Auzel [1] the interest in UC phenomena grew significantly around the year 1990, see Fig. 1. Then near infrared laser diodes became readily available as excitation sources and supplemented the Ti-sapphire laser which is often used in UC research. The research interest was focused towards visible light emission by UC, but soon the rapid development of light emitting diodes dominated the field. A second major upturn in the number of UC publications occurred around the year 2005. It is due to nanomaterials which contribute about half to the $1500 \mathrm{UC}$ publications in 2015. The main research interest is nowadays on nanomaterials [2-4] and biomedical imaging [5-7], but UC is also important in harvesting sub-band-gap photons in solar cells [8], sensor applications [9], or anti-counterfeit applications.

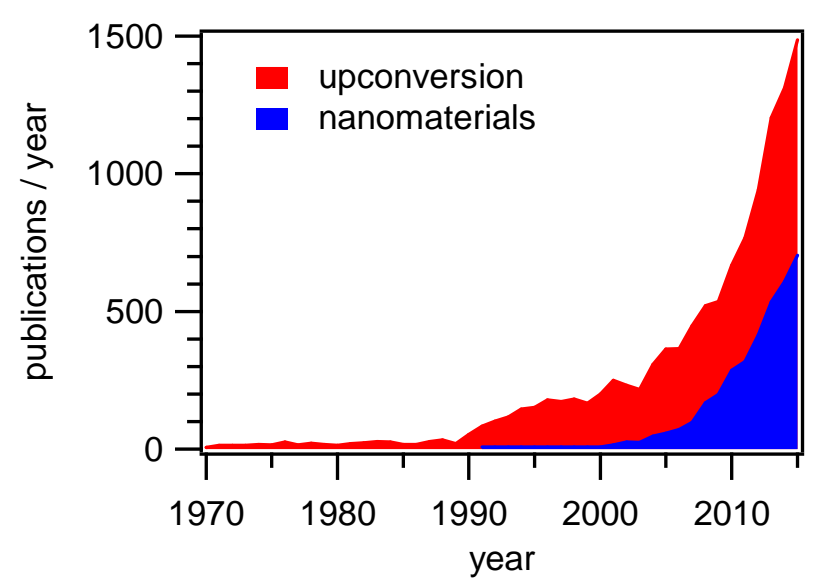

Fig. 1: Annual statistics on upconversion publications as retrieved from SciFinder (ACS) in 02/2016.

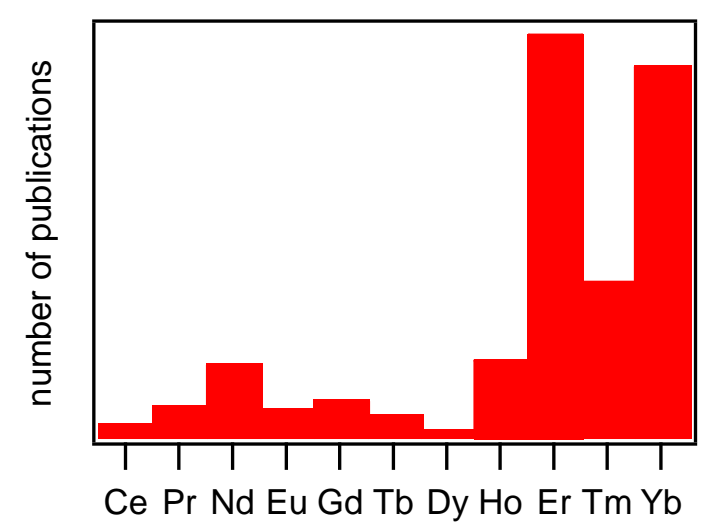

Fig. 2: Relative abundance of rare earth ions in upconversion literature as retrieved from SciFinder (ACS) in 02/2016.

Rare earth (RE) ions dominate in UC research due to the plethora of their metastable emitting energy levels. Spin flip transitions in d-metal ions can also be involved in UC mechanisms [10], as well as the triplet UC in organic molecules [11]. In the following we will focus on upconversion in rare earth ions. Fig. 2 gives an overview on the relative abundance of $\mathrm{RE}^{3+}$ ions in $\mathrm{UC}$ literature. $\mathrm{Er}, \mathrm{Yb}-\mathrm{Er}$, and $\mathrm{Yb}-\mathrm{Tm}$ doped materials 
dominate the field followed by $\mathrm{Ho}$ and $\mathrm{Nd}$. Some $\mathrm{RE}^{3+}$ ions, such as $\mathrm{Ce}$ or $\mathrm{Gd}$, are not involved in an UC mechanism directly but can be used for energy transfer or light emission of UC phosphors.

\section{Upconversion mechanisms}

A luminescence mechanism can be divided into four steps, see Fig. 3a: an excitation step (1), followed by relaxation or energy migration in the excited state (2), the emission of a photon (3), and finally a relaxation in the ground state (4). The energy of the excitation photon $E_{e x}$ is partially emitted as a photon with energy $E_{e m}$ and the energy difference converted into lattice vibrations (heat) with $E_{e x}>E_{e m}$, accordingly. The opposite holds for an upconversion process where the energy of the individual excitation photons is smaller than that of the emitted photon, $E_{\mathrm{ex}}<\mathrm{E}_{\mathrm{em}}$, but several photons are involved in the UC excitation.

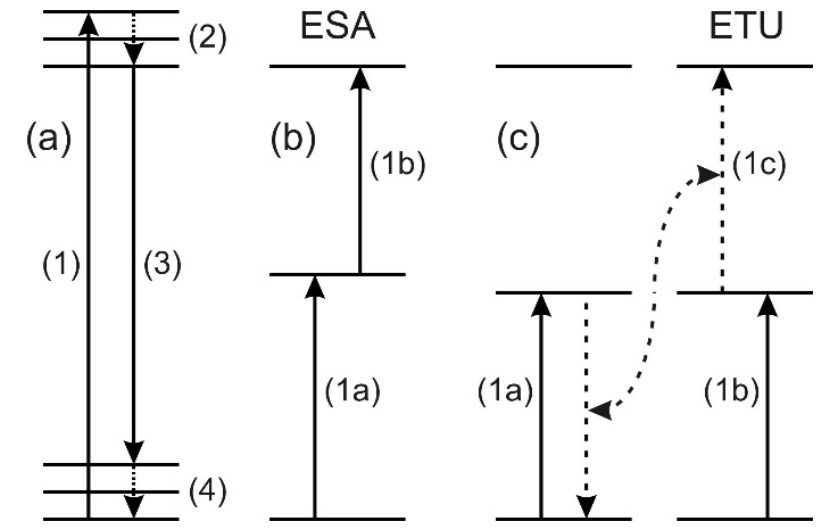

Fig. 3: Schemes for (a) luminescence after one-step excitation, (b) excited state absorption (ESA), and (c) energy transfer upconversion (ETU). (b) and (c) are shown for the most straightforward case of a two-step excitation.

Several UC mechanisms are discussed in literature [1,10]. Among them excited state absorption (ESA) and energy transfer upconversion (ETU) have a broader impact and the following discussion will be restricted to those.

UC luminescence mechanisms can be modelled by systems of rate equations [12-14] which contain the excitation, energy transfer, and emission processes among the energy levels. The absorption and emission are proportional to the oscillator strength of the transition and the excitation density. The energy transfer depends on the concentration of the dopant ions and the spectral overlap of the transitions, i.e. the resonance between the excitation source and the absorption line, or the donor and acceptor levels.

The internal UC quantum efficiency is defined by the number of emitted UC photons divided by the number of absorbed photons [8]. For an UC process, it is intrinsically smaller than $1 / 2$ since one UC photon is emitted after the absorption of two (or more) excitation photons. Since the total efficiency is the product of the efficiencies of all individual steps, the total efficiency is declining for an increasing number of steps involved in a process. 


\section{Experimental}

For Figures 1 and 2, the statistics on UC publications was retrieved using SciFinder (ACS) in February 2016.

A crystal of $\mathrm{LaCl}_{3}: 1 \% \mathrm{Er}^{3+}$ was grown by the Bridgman technique from high-purity, oxygen-free starting materials. Details of the synthesis were published in Ref. [15]. Powder samples of $\beta$-NaYF $4: 20 \% \mathrm{Er}^{3+}$ were synthesized according to Ref. [16]. Details on the crystal structure, the microscopic disorder, and the $\mathrm{RE}^{3+}$ sites are discussed in Ref. [17].

Absorption spectra were measured on Cary $5 \mathrm{e}$ and Cary 6000 spectrometers. UC spectra were excited using Spectra Physics 3900S Ti-sapphire lasers pumped by Millenia Xs frequency doubled $\mathrm{Nd}: \mathrm{YVO}_{4}$ or $\mathrm{Ar}^{+}$ion lasers. The sample was cooled in an Oxford bath cryostat. The luminescence was measured by a Spex double monochromator (500 nm blazed with 1200 grooves $/ \mathrm{mm}$ ), a cooled RCA C31034 photomultiplier, and a Stanford research SR400 photon counting system. For broad band excitation an Oriel $250 \mathrm{~W}$ tungsten lamp was used. The light was focused on the sample by an $\mathrm{f}=5 \mathrm{~cm}$ lens. A set of Si- and dielectric filters (long pass $1300 \mathrm{~nm}$, FEL1300, Thorlabs) blocked the excitation light for shorter wavelengths than $1300 \mathrm{~nm}$. The total UC emission was measured by a Si-detector.

\section{Results and Discussion}

\section{Excited state absorption}

Excited state absorption upconversion takes place on a single ion, see Fig. 3b, and is therefore the most prominent UC process for low dopant concentrations, typically smaller than $1 \%$. The ground state absorption (1a) populates an intermediate level which should have a long life time to facilitate the second absorption step. Excited state absorption (1b) populates the upper level from which the relaxation process starts, cf. Fig. 3a (2-4). The efficiency of the UC process is determined by the oscillator strengths and excitation densities for steps (1a) and (1b), see Fig. 3b. The excitation density depends on the spectral overlap between the excitation profile(s) and the absorption lines. The excitation profile can vary significantly from sub-wavenumber width for laser excitation to broad band for sun light excitation, as will be discussed below. The absorption line width of Stark transitions of rare earth ions in crystalline host lattices is typically in the order of a few wavenumbers.

For one-color laser excitation, the laser wavelength will be adjusted to the absorption maximum of one step, usually the ground state absorption, but it will be off-resonant for the other step in general. The off-resonance can be matched by lattice vibrations (phonons) but the efficiency of this step will be significantly lower than for the resonant step. A two-color two-laser excitation will obviously be the best solution for this problem. Then each laser can be resonant with one excitation step and the optimum efficiency for the ESA process is achieved. 


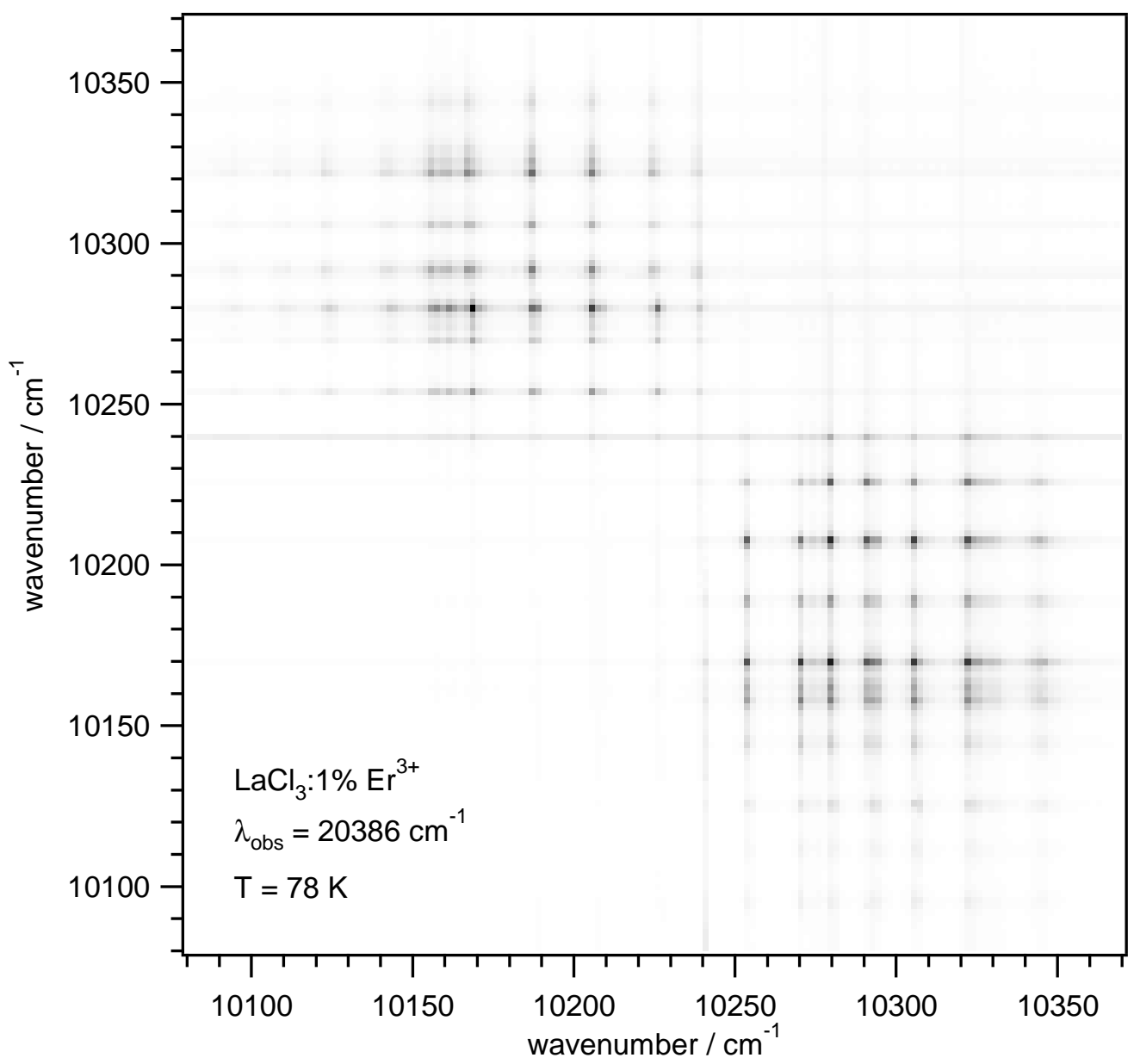

Fig. 4: Two-color two-laser excitation spectrum of $\mathrm{LaCl}_{3}: 1 \% \mathrm{Er}^{3+}$ at $78 \mathrm{~K}$ for $\sigma$ polarization $(E \|$ a) of the excitation light. The intensity of the upconversion luminescence of the $\left.{ }^{4} \mathrm{~F}_{7 / 2}(2) \rightarrow{ }^{4}\right|_{15 / 2}(5)$ transition at $20386 \mathrm{~cm}^{-1}$ is shown on a linear grey scale.

The importance of the resonance problem is demonstrated in Fig. 4. It shows an image plot of two-color excitation spectra of the $\left.{ }^{4} \mathrm{~F}_{7 / 2} \rightarrow{ }^{4}\right|_{15 / 2} \mathrm{UC}$ luminescence for a crystal of $\mathrm{LaCl}_{3}: 1 \% \mathrm{Er}^{3+}$ at $78 \mathrm{~K}$ [15]. Both excitation laser were $\sigma$-polarized (E II a) and carefully aligned for beam overlap in the sample. For each line in Fig. 4, one laser was kept a fixed energy and the other scanned between $10080 \mathrm{~cm}^{-1}$ and $10370 \mathrm{~cm}^{-1}$ resulting in a two-color excitation spectrum of $2 \mathrm{~cm}^{-1}$ resolution. The luminescence is shown on a linear grey scale with an overall maximum of $1 \cdot 10^{6}$ photons/s at excitation energies of $10169 \mathrm{~cm}^{-1}$ and $10280 \mathrm{~cm}^{-1}$. The energies of the $\mathrm{Er}^{3+}$ Stark levels in $\mathrm{LaCl}_{3}$ were previously characterized in detail [15]. All peaks in Fig. 4 can unambiguously be attributed to specific transitions between Stark levels due to their energy and polarization. The mechanism for this ESA process is depicted in Fig. 5a. The transitions of the $\left.\left.{ }^{4}\right|_{15 / 2} \rightarrow{ }^{4}\right|_{11 / 2}$ ground state absorption are located between $10090 \mathrm{~cm}^{-1}$ and 10240 $\mathrm{cm}^{-1}$ whereas those of the $\left.{ }^{4}\right|_{11 / 2} \rightarrow{ }^{4} F_{7 / 2}$ excited state absorption are found between $10240 \mathrm{~cm}^{-1}$ and $10350 \mathrm{~cm}^{-1}$. Due to the low phonon energy of the $\mathrm{LaCl}_{3}$ host lattice, UC luminescence is mainly observed from the ${ }^{4} \mathrm{~F}_{7 / 2}$ state. The intensity of the blue emission of the $\left.{ }^{4} \mathrm{~F}_{7 / 2}(2) \rightarrow{ }^{4}\right|_{15 / 2(5)}$ transition at $20386 \mathrm{~cm}^{-1}$ is shown in Fig. 4. In contrast, most other chlorides show green UC emission from the ${ }^{2} \mathrm{H}_{11 / 2}$ and ${ }^{4} \mathrm{~S}_{3 / 2}$ states which are populated by multi phonon relaxation from the ${ }^{4} \mathrm{~F}_{7 / 2}$ state $[18,19]$. 

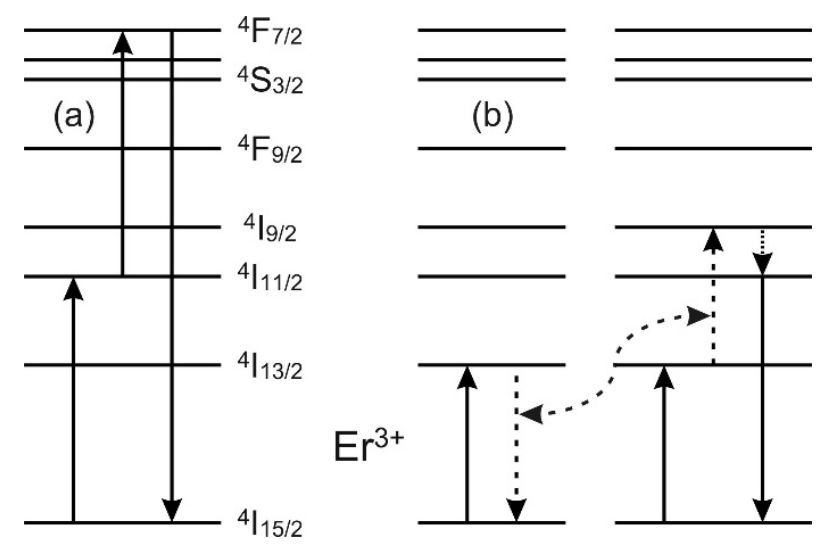

Fig. 5: Energy level scheme of $\mathrm{Er}^{3+}$ with mechanisms for (a) ESA excitation at $980 \mathrm{~nm}$ and (b) ETU excitation at $1.5 \mu \mathrm{m}$.

The diagonal trace of Fig. 4, where both excitation energies are equal, corresponds to the one-color excitation spectrum. It shows a local maximum of $1 \cdot 10^{5}$ photons $/ \mathrm{s}$ at $10240 \mathrm{~cm}^{-1}$, see the black trace in Fig. 6 . Here, an accidental resonance between the ${ }^{4} I_{15 / 2}(1) \rightarrow{ }^{4} I_{11 / 2}(6)$ and ${ }^{4} I_{11 / 2}(6) \rightarrow{ }^{4} F_{7 / 2}(1)$ transitions occurs. The resonance results in a maximum of the one-color spectrum despite the oscillator strengths of the involved transitions are by far not the strongest within the respective bands. Also, the Boltzmann population of the ${ }^{4} l_{11 / 2}(6)$ level is lowest within this state. Accordingly, the off-diagonal intensities in Fig. 4, resulting from excitation by two different energies, are much higher than for one-color excitation.

The strongest transitions within the bands are revealed by two-color excitation spectra, see the red and blue traces in Fig. 6. Keeping one excitation energy fixed at a maximum of one band, the second laser is scanned and probes the absorption spectrum of the other band. In this way the overall maxima are reliably determined. For $\sigma$-polarization, they correspond to $10169 \mathrm{~cm}^{-1}$ and $10280 \mathrm{~cm}^{-1}$ for the $\left.\left.{ }^{4}\right|_{15 / 2} \rightarrow{ }^{4}\right|_{11 / 2}$ and $\left.{ }^{4}\right|_{11 / 2} \rightarrow{ }^{4} \mathrm{~F}_{7 / 2}$ transitions, respectively. For $\pi$-polarization, the best excitation energies are $10226 \mathrm{~cm}^{-}$ ${ }^{1}$ and $10306 \mathrm{~cm}^{-1}$, see Fig. 7, and the overall maximum luminescence intensity of $2 \cdot 10^{6}$ photons/s is even higher. The one-color excitation spectrum in $\pi$-polarization shows lower luminescence intensities than its counterpart in $\sigma$-polarization, cf. Figs. 6-7, because no accidental resonance occurs.

Thus, significantly higher luminescence intensities are obtained by two-color resonant excitation compared to one-color excitation for the same total excitation density. In our example the UC luminescence intensity increased 20-times between one- and twocolor experiments. 


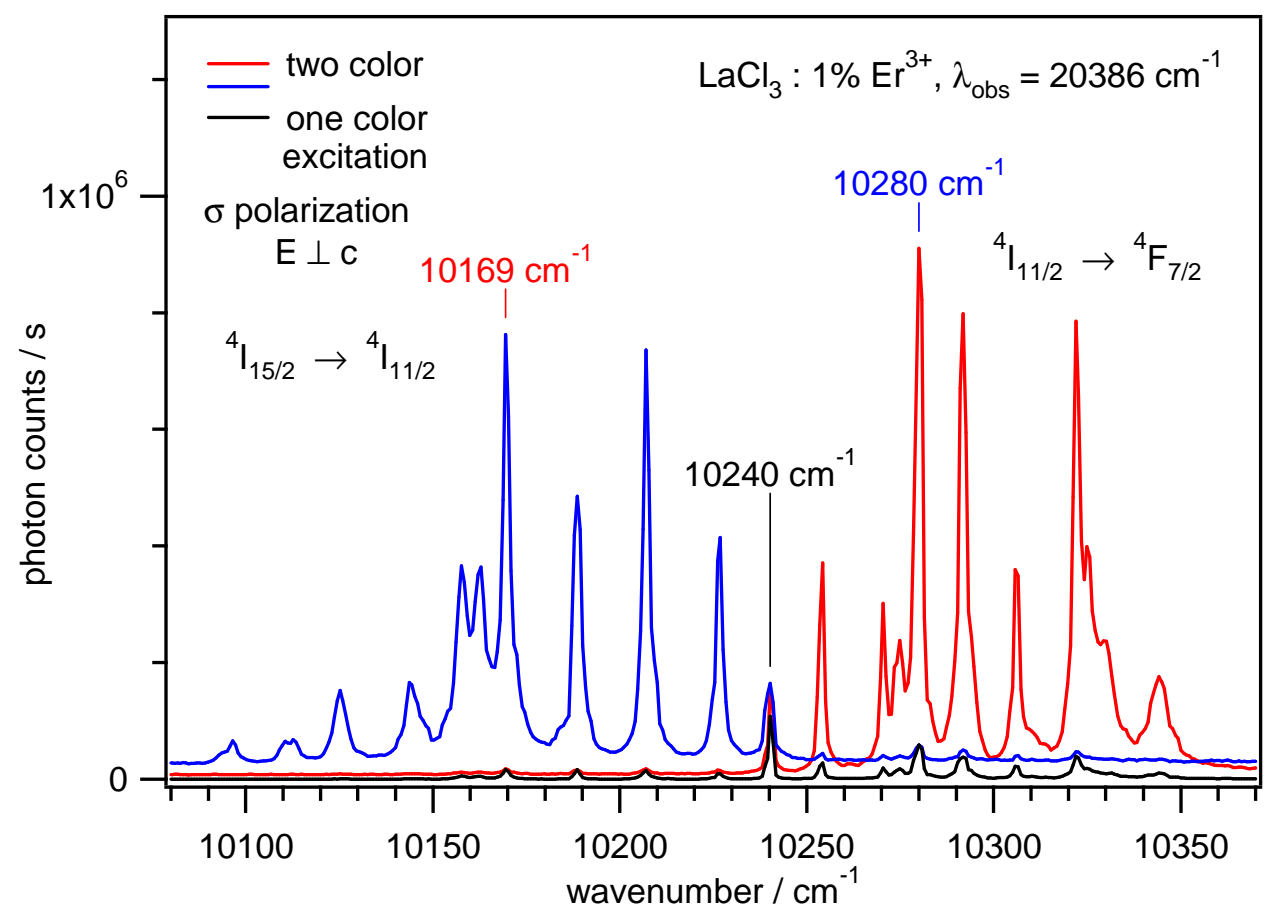

Fig. 6: One- and two-color excitation spectra of $\mathrm{LaCl}_{3}: 1 \% \mathrm{Er}^{3+}$ at $78 \mathrm{~K}$ for $\sigma-$ polarization $(\mathrm{E} \perp \mathrm{c})$ of the excitation light. The one-color spectrum (black trace) corresponds to the diagonal of Fig. 4. The two-color spectra (blue and red traces) reveal the overall emission maximum at $10169 \mathrm{~cm}^{-1}$ and $10280 \mathrm{~cm}^{-1}$ excitation energies.

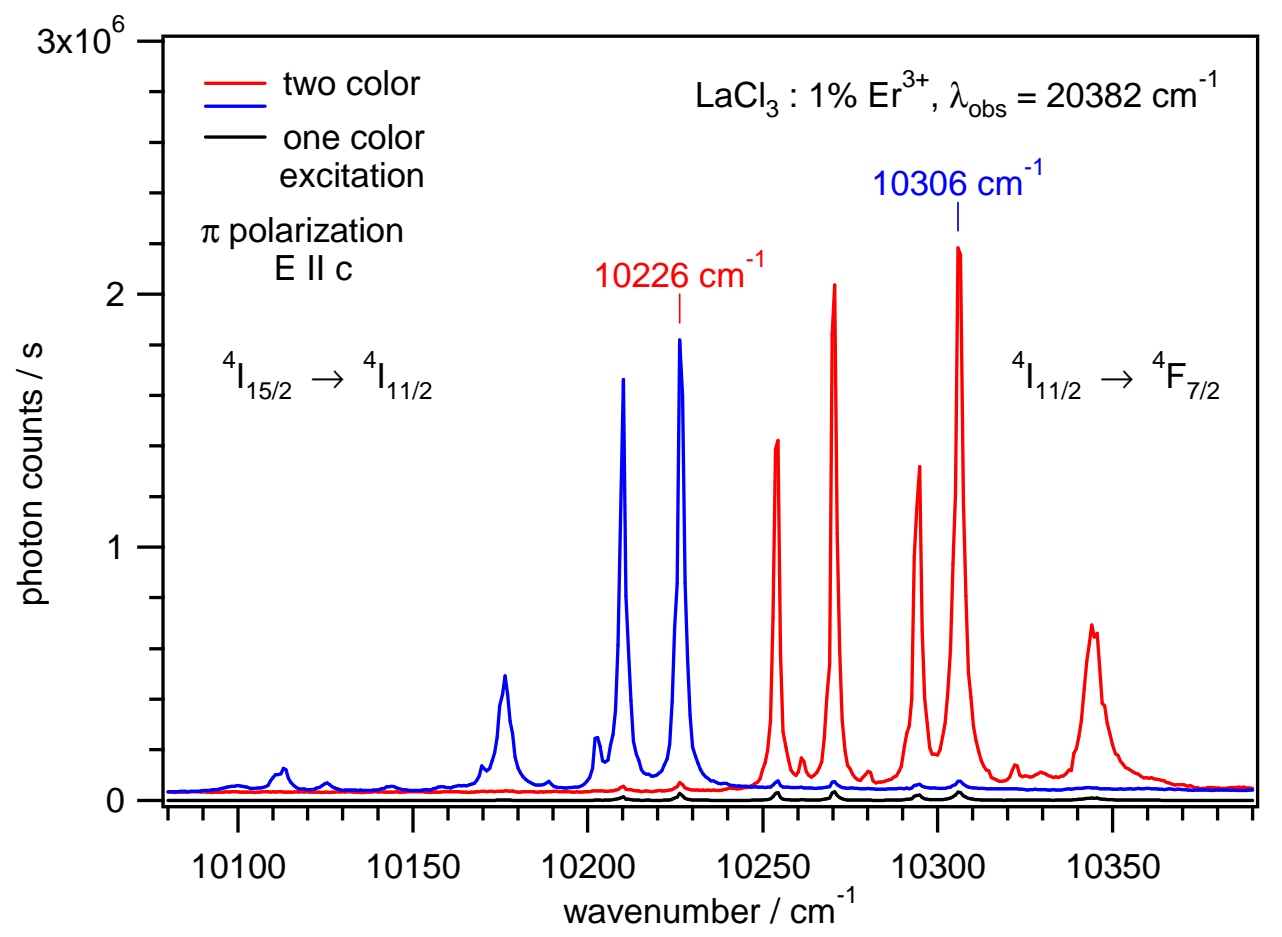

Fig. 7: One- and two-color excitation spectra of $\mathrm{LaCl}_{3}: 1 \% \mathrm{Er}^{3+}$ at $78 \mathrm{~K}$ for $\pi$-polarization $(E \| \mathrm{C})$ of the excitation light. The one-color spectrum (black trace) has a low intensity. The two-color spectra (blue and red traces) demonstrate the overall emission maximum at $10226 \mathrm{~cm}^{-1}$ and $10306 \mathrm{~cm}^{-1}$ excitation energies. 
One- and two-color UC luminescence spectra show characteristic power dependencies, see Fig. 8. For one-color excitation a slope of two was found which is expected for a two-step UC process in the low power regime [12]. On the other hand, for two-color excitation the luminescence has a linear power dependence for each color if the excitation bands are separated in energy. This is in good agreement with the experiment where a slope of $0.84(3)$ was found for $\mathrm{LaCl}_{3}: 1 \% \mathrm{Er}^{3+}$ at $78 \mathrm{~K}$. The power for the $\left.{ }^{4}\right|_{15 / 2} \rightarrow{ }^{4} I_{11 / 2}$ excitation at $10169 \mathrm{~cm}^{-1}$ was varied and kept fixed at $10280 \mathrm{~cm}^{-1}$ for the ${ }^{4} \mathrm{I}_{11 / 2} \rightarrow{ }^{4} \mathrm{~F}_{7 / 2}$ excitation, cf. Fig. 6 .

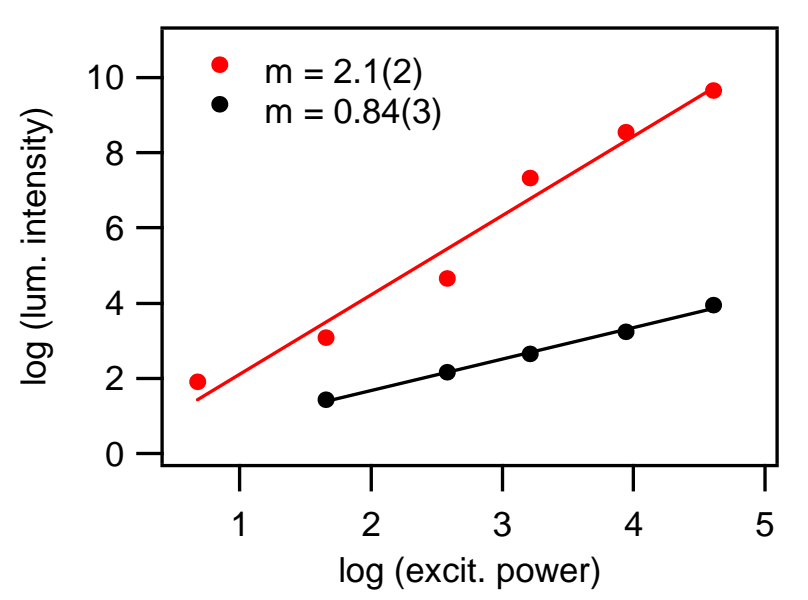

Fig. 8: Power dependence of the UC luminescence of $\mathrm{LaCl}_{3}: 1 \% \mathrm{Er}^{3+}$ at $78 \mathrm{~K}$ for one color (red dots) and two color (black dots) excitation. For two-color excitation the power at $10169 \mathrm{~cm}^{-1}$ was varied and kept fixed at $10280 \mathrm{~cm}^{-1}$, cf. Fig. 6 . The slope (m) was obtained from linear fits to the data (red and black lines).

\section{Energy transfer upconversion}

Broad-band excited upconversion has attracted significant interest within the last decade. This research was motivated by the aim to improve the efficiency of Si solar cells by upconversion of sub-band-gap photons. Since UC processes are often limited by the available excitation power such an aim appears out of reach on a first view. But within recent years significant enhancements have been achieved [8]. Only the most efficient upconversion processes yield a significant contribution for low power excitation which puts the focus on two-step ETU. From a technical point of view, an upconverter material is attached to the back side of a Si solar cell and preferably operated in a solar concentrator module [8].

In our search for a suitable material for this application we investigated possible upconverter ions. $\beta-\mathrm{NaYF}_{4}$ was chosen as host lattice since $\beta-\mathrm{NaYF}_{4}: \mathrm{Yb}, \mathrm{Er}$ and $\beta$ $\mathrm{NaYF}_{4}: \mathrm{Yb}, \mathrm{Tm}$ are well known as very efficient green and blue emitting UC phosphors, respectively $[16,20]$. Powder samples doped with $20 \% \mathrm{RE}^{3+}$ ions were prepared. They were excited by broad-band near-infrared light from a W-lamp. The wavelengths longer than $1300 \mathrm{~nm}$ were separated by Si and long-pass filters and focused on the sample. The upconversion emission from the sample was measured by a Si-detector. The results are shown in Fig. 9. The UC luminescence from $\mathrm{Er}$ is more than two orders of 
magnitude stronger than that from $\mathrm{Pr}, \mathrm{Nd}, \mathrm{Sm}$, and $\mathrm{Tm}$. The signal from $\mathrm{Ho}$ is even weaker and Dy at the detection limit of the apparatus. The huge difference between $\mathrm{Er}$ and the other dopant ions proves the extraordinary performance of the $\left.\left.{ }^{4}\right|_{15 / 2} \rightarrow^{4}\right|_{13 / 2}$ ETU and rules out the others ions for efficient broad band excitation in this spectral range.

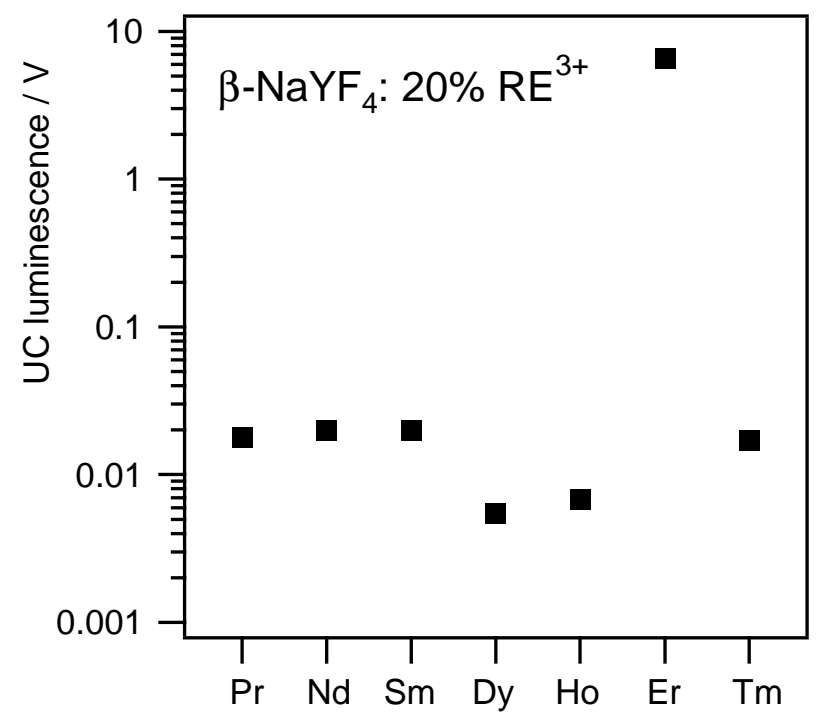

Fig. 9: Upconversion luminescence of $\beta-\mathrm{NaYF}_{4}: 20 \% \mathrm{RE}^{3+}$ powder samples for subSi-band-gap broad-band infrared excitation by a W-lamp.

The UC mechanism is shown in Fig. $5 b$. The $\left.\left.{ }^{4}\right|_{15 / 2} \rightarrow{ }^{4}\right|_{13 / 2}$ absorption around $1.5 \mu \mathrm{m}$ is electric and magnetic dipole allowed and has a high oscillator strength. It is comparable to the ${ }^{2} \mathrm{~F}_{7 / 2} \rightarrow{ }^{2} \mathrm{~F}_{5 / 2}$ absorption of $\mathrm{Yb}^{3+}$ which is often used as UC sensitizer. In the next step, energy is transferred between two neighboring $\mathrm{Er}^{3+}$ ions both in the $\left.{ }^{4}\right|_{13 / 2}$ excited state. This ETU promotes one ion to the $\left.{ }^{4}\right|_{9 / 2}$ state whereas the other returns to the ground state. Multi phonon relaxation to the $\left.{ }^{4}\right|_{11 / 2}$ state rapidly depopulates the $\left.{ }^{4}\right|_{9 / 2}$ state and prohibits losses by cross relaxation, i.e. the reverse process of ETU. From the ${ }^{4} l_{11 / 2}$ state (or higher states) energy can be transferred to $\mathrm{Si}$ and thereby increase the efficiency of a solar cell by upconversion.

The broad-band excitation has a low excitation density, as compared to a laser, but it overcomes the resonance problem which was discussed above for ESA. All wavelengths are available in the broad-band excitation spectrum and every UC step is resonantly excited.

The absorption spectrum of the $\left.\left.{ }^{4}\right|_{15 / 2} \rightarrow{ }^{4}\right|_{13 / 2}$ transition of $\beta-\mathrm{NaYF}_{4}: 20 \% \mathrm{Er}^{3+}$ is shown in Fig. 10b. It has a maximum at $1523 \mathrm{~nm}\left(6566 \mathrm{~cm}^{-1}\right)$ and a width of about $100 \mathrm{~nm}$. The UC luminescence for this excitation is shown in Fig. 11. The by far strongest emission is observed from the $\left.{ }^{4}\right|_{11 / 2}$ state. Its fraction compared to the total UC luminescence can be as high as $98 \%$ and mainly depends on the $\mathrm{Er}^{3+}$ concentration and the excitation density.

Fig. 10a shows the excitation spectrum of the optical efficiency of a Si solar cell due to upconversion by two-step ETU as discussed above. This spectrum closely resembles the $\left.\left.{ }^{4}\right|_{15 / 2} \rightarrow{ }^{4}\right|_{13 / 2}$ absorption spectrum shown in Fig. 10b. It demonstrates an important difference between ETU and ESA. For ETU the excitation spectrum depends on the 
ground state absorption of the upconverter ion (or the sensitizer, e.g. $\mathrm{Yb}^{3+}$ ) but it contains no features due to the energy transfer steps. For ESA on the other hand, the excitation spectrum is a convolution of the absorption spectra of the individual steps, cf. Figs. 6-7.
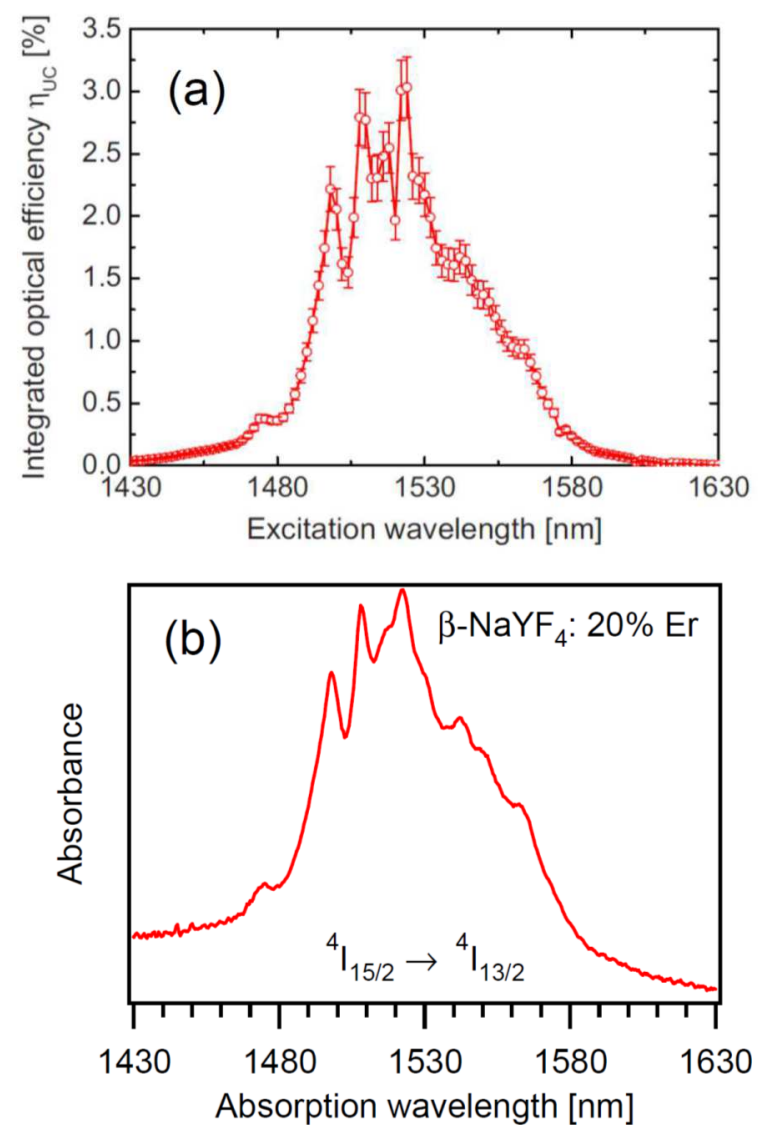

Fig. 10: (a) Integrated optical efficiency of a Si solar cell due to upconversion of the $\left.\left.{ }^{4}\right|_{15 / 2} \rightarrow{ }^{4}\right|_{13 / 2}$ excitation of $\mathrm{Er}^{3+}$ in $\beta-\mathrm{NaYF}_{4}: 20 \% \mathrm{Er}$, reproduced from [21]. (b) Absorption spectrum of the $\left.{ }^{4} I_{15 / 2} \rightarrow{ }^{4}\right|_{13 / 2}$ transition in $\beta-\mathrm{NaYF}_{4}: 20 \% \mathrm{Er}$.

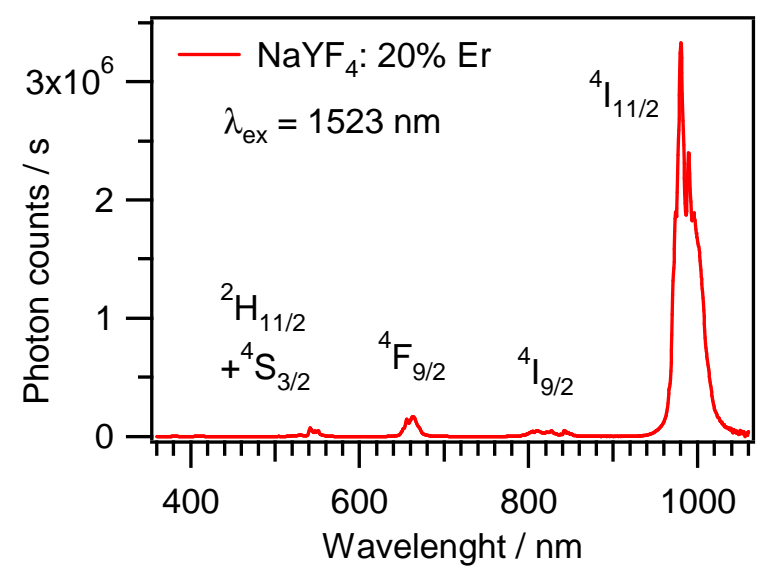

Fig. 11: UC luminescence spectrum of $\beta-\mathrm{NaYF}_{4}: 20 \% \mathrm{Er}$ for $\left.\left.{ }^{4}\right|_{15 / 2} \rightarrow{ }^{4}\right|_{13 / 2}$ excitation. The initial states of the luminescence bands to the ground state are shown in the graph. 


\section{Summary}

Upconversion processes show characteristic fingerprints which can be used to identify their mechanisms. Excitation spectra for ESA are a convolution of the absorption spectra of the individual steps, whereas they resemble the ground state absorption for ETU. The UC luminescence intensity shows a linear dependence for each excitation energy for multi-color ESA. For one-color ESA the exponent depends on the number of excitation steps, as it is also the case for ETU. All of that applies for the low power limit. For higher power densities, saturation effects occur and significantly reduce the exponents.

UC luminescence is not only observed for laser excitation. Highly efficient two-step ETU processes, as the $\left.\left.{ }^{4}\right|_{15 / 2} \rightarrow{ }^{4}\right|_{13 / 2}$ excitation in $\beta-\mathrm{NaYF}_{4}: 20 \% \mathrm{Er}$, give rise to a strong UC luminescence already for low-power broad-band excitation by a lamp or the sun.

\section{Acknowledgements}

The synthesis of UC phosphor powder samples by D. Biner is gratefully acknowledged, as well as fruitful discussions with P. Villanueva. Financial support was obtained from the Swiss National Science Foundation and the European Union FP7 program via the Nanospec (246200) and Luminet (316906) projects. 


\section{References}

[1] F. Auzel, Upconversion and Anti-Stokes Processes with $f$ and $d$ lons in Solids, Chem. Rev. 104 (2004) 139-173, doi:10.1021/cr020357g.

[2] M. Haase and H. Schäfer, Upconverting Nanoparticles, Angew. Chem. Int. Ed. 50 (2011) 5808-5829, doi:10.1002/anie.201005159.

[3] X. Li, F. Zhang, and D. Zhao, Highly efficient lanthanide upconverting nanomaterials: Progresses and challenges, Nano Today 8 (2013) 643-676, doi:10.1016/j.nantod.2013.11.003.

[4] D. J. Gargas, E. M. Chan, A. D. Ostrowski, S. Aloni, M. V. P. Altoe, E. S. Barnard, B. Sanii, J. J. Urban, D. J. Milliron, B. E. Cohen, and P. J. Schuck, Engineering bright sub-10-nm upconverting nanocrystals for single-molecule imaging, Nature Nanotechnology 9 (2014) 300-305, doi:10.1038/nnano.2014.29.

[5] J. Zhou, Z. Liu, and F. Li, Upconversion nanophosphors for small-animal imaging, Chem. Soc. Rev. 41 (2012) 1323-1349, doi:10.1039/c1cs15187h.

[6] S. Gai, C. Li, P. Yang, and J. Lin, Recent Progress in Rare Earth Micro/Nanocrystals: Soft Chemical Synthesis, Luminescent Properties, and Biomedical Applications, Chem. Rev. 114 (2014) 2343-2389, doi:10.1021/cr4001594.

[7] W. Zheng, P. Huang, D. Tu, E. Ma, H. Zhuab, and X. Chen, Lanthanide-doped upconversion nano-bioprobes: electronic structures, optical properties, and biodetection, Chem. Soc. Rev. 44 (2015) 1379-1415, doi: 10.1039/c4cs00178h.

[8] J. C. Goldschmidt and S. Fischer, Upconversion for Photovoltaics - a Review of Materials, Devices and Concepts for Performance Enhancement, Adv. Optical Mater. 3 (2015) 510-535, doi:10.1002/adom.201500024.

[9] L. H. Fischer, G. S. Harms, and O. S. Wolfbeis, Upconverting Nanoparticles for Nanoscale Thermometry, Angew. Chem. Int. Ed. 50 (2011) 4546-4551, DOI:10.1002/anie.201006835.

[10] D. R. Gamelin and H. U. Güdel, Upconversion Processes in Transition Metal and Rare Earth Metal Systems, Top. Curr. Chem. 214 (2001) 1-56.

[11] Y. C. Simon and C. Weder, Low-power photon upconversion through triplettriplet annihilation in polymers, J. Mater. Chem. 22 (2012) 20817-20830, doi: 10.1039/c2jm33654e.

[12] M. Pollnau, D. Gamelin, S. R. Lüthi, H. U. Güdel, and M. Hehlen, Power Dependence of Upconversion Luminescence in Lanthanide and Transition-Metal-Ion Systems, Phys. Rev. B 61 (2000) 3337-3346.

[13] J. Suyver, A. Aebischer, S. García-Revilla, P. Gerner, and H. U. Güdel, Anomalous Power Dependence of Sensitized Upconversion Luminescence. Phys. Rev. B 71 (2005) 125123.

[14] P. Villanueva-Delgado, K. W. Krämer, and Rafael Valiente, Simulating Energy Transfer and Upconversion in $\beta$-NaYF 4 : $\mathrm{Yb}^{3+}, \mathrm{Tm}^{3+}$, J. Phys. Chem. C 119 (2015) 23648-23657, doi:10.1021/acs.jpcc.5b06770. 
[15] K. W. Krämer, H. U. Güdel, and R. N. Schwartz, Infrared-to-visible upconversion in $\mathrm{LaCl}_{3}: 1 \% \mathrm{Er}^{3+}$. Energy-level and line-strength calculations, Phys. Rev. B 56 (1997) 13830-13840.

[16] K. W. Krämer, D. Biner, G. Frei, H. U. Güdel, M. P. Hehlen, and S. R. Lüthi, Hexagonal sodium yttrium fluoride based green and blue emitting upconversion phosphors, Chem. Mater. 16 (2004) 1244-1251.

[17] A. Aebischer, M. Hostettler, J. Hauser, K. W. Krämer, T. Weber, H. U. Güdel, and H.B. Bürgi, Structural and spectroscopic characterization of the active sites in a family of light-emitting sodium lanthanide tetrafluorides, Angew. Chem. Int. Ed. 45 (2006) 2802-2806.

[18] K. Krämer and H. U. Güdel, Upconversion luminescence in $\mathrm{K}_{2} \mathrm{LaX}_{5}: \mathrm{Er}^{3+}(\mathrm{X}=\mathrm{Cl}$, Br), J. Alloys Comp. 207/208 (1994) 128-132.

[19] M. P. Hehlen, K. Krämer, H. U. Güdel, R. A. McFarlane, and R. N. Schwartz, Upconversion in $\mathrm{Er}^{3+}$ dimer systems: Trends within the series $\mathrm{Cs}_{3} \mathrm{Er}_{2} \mathrm{X}_{9}(\mathrm{X}=\mathrm{Cl}, \mathrm{Br}, \mathrm{I})$, Phys. Rev. B 49 (1994) 12475-12484.

[20] T. Kano, H. Yamamoto, Y. Otomo, NaLnF4: $\mathrm{Yb}^{3+}, \mathrm{Er}^{3+}(\mathrm{Ln}$ : Y, Gd, La): Efficient Green-Emitting Infrared-Excited Phosphors, J. Electrochem. Soc. 119 (1972) 15611564, http://dx.doi.org/10.1149/1.2404042.

[21] S. Fischer, J. C. Goldschmidt, P. Löper, G. H. Bauer, R. Brüggemann, K. Krämer, D. Biner, M. Hermle, and S. W. Glunz, Enhancement of silicon solar cell efficiency by upconversion: Optical and electrical characterization, J. Appl. Phys. 108 (2010) 044912, 1-11, doi:10.1063/1.3478742. 


\section{Highlights}

The crucial importance of resonance is discussed for one- and two-color excitation spectra of the ${ }^{4} \mathrm{~F}_{7 / 2}$ upconversion luminescence in $\mathrm{LaCl}_{3}: 1 \% \mathrm{Er}^{3+}$.

The upconversion luminescence from broad-band excitation of the $\left.\left.{ }^{4}\right|_{15 / 2} \rightarrow{ }^{4}\right|_{13 / 2}$ transition in $\beta-\mathrm{NaYF}_{4}: 20 \% \mathrm{Er}$ is very efficient for the excitation of Si solar cells.

The excitation spectra and power dependences for ESA and ETU upconversion processes are discussed. 